\title{
Study on Creep Characteristics of Cemented Waste Rock Backfills
}

\author{
Changhong $\mathrm{Li}^{1,2}$, Qiantian $\mathrm{Ma}^{1,2, *}$, Guoliang $\mathrm{Chen}^{3}$ and Hai Shao ${ }^{4}$
}

\author{
${ }^{I}$ Key Laboratory of the Ministry of Education of China for High-efficient Mining and Safety of Metal Mines, University \\ of Science and Technology Beijing, Beijing, China; ${ }^{2}$ School of Civil and Environmental Engineering, University of Sci- \\ ence and Technology Beijing, Beijing, China; ${ }^{3}$ Ganzhou rare earth mineral industry CO.LTD, Ganzhou, China; \\ ${ }^{4}$ Technical Center for Geo-hazards Emergency of MLR, Beijing, China
}

\begin{abstract}
Slip instability and tensor instability are two kinds of failure modes of cemented waste rock backfills. In order to simulate the creep characteristics of cemented waste rock backfills, PFC was carried out. The results show that: rocks inside the models will have displacement under load. The expansion is caused by large lateral displacement of the rocks. A non-stationary creep model of cemented waste rock backfills was established based on the results of simulation. This model can be used to describe the whole creep characteristics of cemented waste rock backfills better.
\end{abstract}

Keywords: Cemented waste rock backfills, non-stationary Nishihara model, PFC, short-time creep.

\section{INTRODUCTION}

Currently, many mines would replace native pillars with artificial pillars to support roof or country rocks in upper wall in order to reduce losses and improve the stoping rate of resources. Considering many negative effects such as waste emissions and environmental degradation brought about by stoping resources, and to save costs, artificial pillars are generally adopted with mining solid waste (mill tailings, waste rocks) as the main ingredient, coupled with the cementing materials to form cemented waste rock backfills. Meanwhile, artificial pillars are the most important support structure for safe stoping of ore body. In the complex external mechanical environment of downhole-stoping, once it is destroyed or instable (such as backfill roof and floor pillars, backfill studding and backfill supporting the upper and lower sides), it will result in the collapse of roof, strata displacement of the upper and lower sides, or even induce the occurrence of deep stoping rock burst, which has become a serious threat to secure stoping. Therefore, the study of mechanical properties and damage mechanism of cemented waste rock backfills in a complex environment lays an important foundation for filing material selection, optimization and stability analysis. On this basis, it is suggested that filling stability monitoring and prediction method is a major technical problem that mines should urgently solve.

In recent years, along with the application and popularization of the cemented filling mining method, cemented waste rock backfills attract great attention as a new kind of engineering material $[1,2]$. Meanwhile, due to the impact of continuous operations in mines, constant load on artificial pillars would alter with the stoping of surrounding ore blocks, making artificial pillars impossible to withstand the effects of a constant constant load for a long time. This is actually a short-term graded creep process. Accordingly, it will be very meaningful to investigate the characteristics of the short-term graded creep of artificial pillars and describe them. In terms of concrete materials, the existing Burgers model, Nishihara model and its improved model [3] depict the rheological characteristics. However, these models are stationary models and fail to completely describe the rheological characteristics of the materials, especially the deformation characteristics in the accelerated creep stage.

Meanwhile, with the rapid development of computer technology, computer simulation has been more and more widely employed in science and engineering, thus making numerical analysis an analytical approach equally important to theoretical analysis and experimental research. Particle flow code (PFC) is a new kind of numerical analysis method. It was established by P. A. Cundall and O. D. Strack by introducing molecular dynamics based on the discrete element method. This theory is focused on using mesomechanics to explain the damage and fracture mechanisms of materials [4]. PFC has been widely used in studying rocks and asphaltlike materials [5-7], but has not been yet applied to the filling materials.

In regard to cemented waste rock backfills, the inoculation, production, evolution, expansion and fracture of microcracks occur between the waste rocks. As thus, different waste rock spatial combinations will lead to different ways of instability. In this paper, a stress corrosion model provided by PFC was used to carry out a creep simulation of the 3D model of cemented waste rock backfills, which further revealed the internal change characteristics of cemented waste rock backfills under the action of load. And on this basis a non-stationary cemented waste rock backfills creep model was established, which was capable of well depicting the whole creep course of cemented waste rock backfills. 


\section{PFC SIMULATION}

\subsection{Selection of Bond Model}

PFC is a program based on the discrete element method. It simulates material particles by establishing spheres, and simulates the cementation between material particles by different bond models. PFC provides two standard bond models: contact bond model and parallel bond model [8]. Wherein, the contact bond model only transfers forces but not torque, so this paper uses the parallel bond model to simulate the creep behavior of the cemented waste rock backfills. Fig. (1) is the principle for the parallel bond model. There is certain area and stiffness bonding in the contact points of the parallel bond model, which can limit the particles' rotation and transmit force and torque. Under the action of load, the stress in parallel bond can be obtained through Formula (1):

$$
\left.\begin{array}{l}
\bar{\sigma}=\frac{-\bar{F}_{i}^{n}}{A_{2}}+\frac{\left|\bar{M}_{i}^{s}\right| \bar{R}}{I} \\
\bar{\tau}=\frac{\left|\bar{F}_{i}^{s}\right|}{A_{2}}+\frac{\left|\bar{M}_{i}^{n}\right| \bar{R}}{J}
\end{array}\right\}
$$

where $A=\pi \bar{R}^{2} ; J=\pi \bar{R}^{4} / 2 ; I=\pi \bar{R}^{4} / 4 ; \bar{R}$ was the average radius for parallel bond contact in the contact point. When the normal stress exceeded $\sigma$ or the tangential stress exceeded $\bar{\tau}$, the parallel bond was damaged, respectively generating tension micro-cracks or shear micro-cracks. Meanwhile, on the basis of the parallel bond model, PFC provides a pressure stress corrosion model (PSC), which can achieve simulation of material creep by continuously reducing the radius $\bar{R}$ of parallel bond [9].

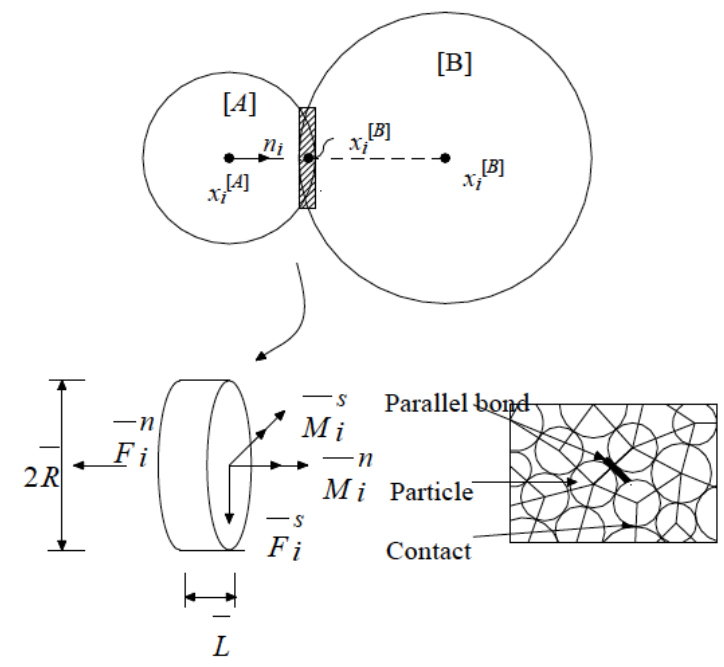

Fig. (1). Parallel bond model.

\subsection{Establishment of Numerical Model}

As shown in Fig. (2), according to the failure mode, cemented waste rock backfill specimens were divided into two types: as shown in Fig. (2a), when waste rocks in the left and right were combined at a relatively large angle (less than $58^{\circ}$ ), slip instability of the specimens would occur between the two parts of waste rock combination. As shown in Fig. (2b), when the waste rocks in the upper and lower side were combined at a relatively small angle (greater than $58^{\circ}$ ), tensor instability of the specimens would occur between the two parts of waste rock combination. Waste rock combination on both sides of centre joints was made of from the cementcemented tailings backfills. The centre joint is the cementtailings backfill located in the middle part of the specimen and penetrates through most part of it.

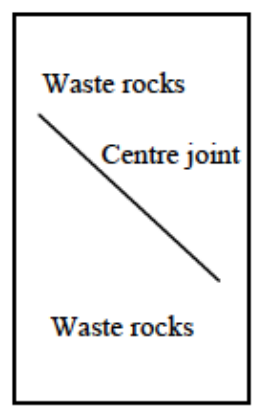

(a) Type I

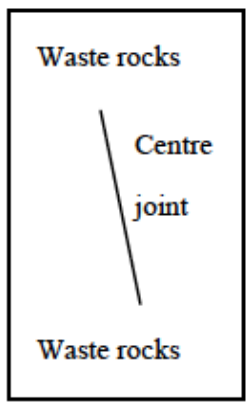

(b) Type II
Fig. (2). Structure in blocky rock cemented fill.

The model style and size of PFC simulation was consistent with the size of the style used in the test, which was $\phi 75 \times 150 \mathrm{~mm}$ cylinders, as shown in Fig. (3a). In other words, in the appearance of cemented waste rock backfills model, the orange ball was cement-tailings backfill particles.

By invoking PFC, clump functions were built and waste rocks were constructed in the model. Consistent to waste rock particle size in the cemented waste rock backfill specimens in indoor trials, it was of irregular polyhedral and the gap between the waste rocks was $5 \sim 10 \mathrm{~mm}$. Figs. (3b and $\mathbf{c})$ show that, in accordance with the center joint angle in the model, two models were established, and their center joint angles were respectively $40^{\circ} \sim 50^{\circ}$ (Type-I corresponded to Type I cemented waste rock backfill specimens) and $60^{\circ} \sim$ $70^{\circ}$ (Type-II corresponded to Type II cemented waste rock backfill specimens). The blue ball was waste rock particles. Since the model was three-dimensional, and the shape of the waste rocks was irregular polyhedron, many joints among waste rocks in Fig. (3) were invisible as blocked by waste rocks in the front. Joints among waste rocks were filled with cement- tailings cement particles.

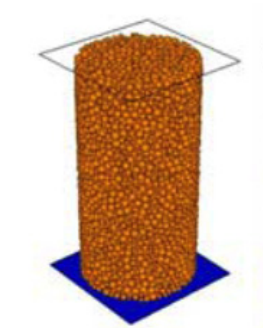

(a)Model appearance

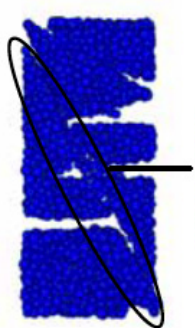

(b) type-I

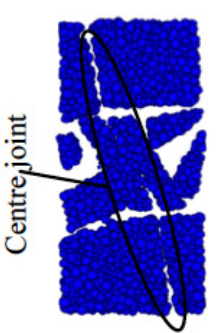

(c) type-II
Fig. (3). Internal and external structure of PFC model. 
The PFC program is to set mesomechanics parameters for the mechanical properties of simulation materials. Literature [10-12] discusses the acquisition methods for the micromechanics parameters in the parallel bond model and the stress corrosion model. In this paper, under the same loading and rate conditions, a matching was conducted between the value of waste rock model and cement-sand to 1:4 cementcemented tailings backfill model under static compression and the value in laboratory tests, resulting in micromechanical parameters of waste rocks and cement-cemented tailings backfills. Also, these micromechanical parameters were respectively given corresponding particles in the cemented waste rock backfill model. Table 1 gives the micromechanical parameters.

Table 1. Micromechanical parameters for different materials.

\begin{tabular}{|c|c|c|}
\hline Parameter & Phyllite particles & $\begin{array}{c}\text { Cement- cemented } \\
\text { tailing particles }\end{array}$ \\
\hline \hline $\begin{array}{c}\text { Particle modulus of } \\
\text { elasticity/GPa }\end{array}$ & 33.5 & 7.0 \\
\hline $\begin{array}{c}\text { Particle stiffness } \\
\text { ratio/ } \mathrm{k}_{\mathrm{n}} / \mathrm{k}_{\mathrm{s}}\end{array}$ & 1.0 & 0.8 \\
\hline $\begin{array}{c}\text { Particle normal bond- } \\
\text { ing strength/KPa }\end{array}$ & 62.5 & 6.0 \\
\hline $\begin{array}{c}\text { Particle tangential } \\
\text { bonding strength/KPa }\end{array}$ & 60 & 5.45 \\
\hline $\begin{array}{c}\text { Friction coefficient of } \\
\text { particle }\end{array}$ & 0.6 & 0.4 \\
\hline $\begin{array}{c}\text { Material parameter } \\
\text { Crack initiation } \\
\text { stress/MPa }\end{array}$ & $6.5 \times 10^{-15}$ & $4 \times 10^{-16}$ \\
\hline
\end{tabular}

\subsection{Simulation Results}

Creep simulation was implemented on the two types of models. Fig. (4) shows the change courses of the internal waste rock structures under the creep load state of the two models. As for Type-I model in the instantaneous load stage before entering the constant load state, although some waste rocks within the model moved, they moved to a small extent. However, after entering into the constant load stage, waste rocks within the model also moved enormously in the accelerated creep stage. Finally, it displayed the instability form as shown in Fig. (4a). As Fig. (4b) shows, in the instantaneous load stage before entering the constant load, some waste rocks in Type-II model have begun to clearly separate. Wherein, the upper waste rocks' separation degree was bigger than the other parts, while the other parts of the waste rocks only dramatically separated in the acceleration creep stage.

Meanwhile, as shown in Fig. (4), when two types of models were conducted creep simulation, displacement monitoring was carried out in waste rocks that have gone through significant lateral displacement in its lower portion. The monitoring method was to carry out displacement monitoring for all balls within the waste rocks and then extract the average value as the displacement of waste rocks as a whole. Fig. (5) demonstrates the monitoring results. As can be seen, there was not much axial displacement of monitored waste rocks in the two models, but the lateral displacement of monitored waste rocks in the Type-II model was much greater than that in the Type-I model, thereby resulting in expansion of the Type-II model.
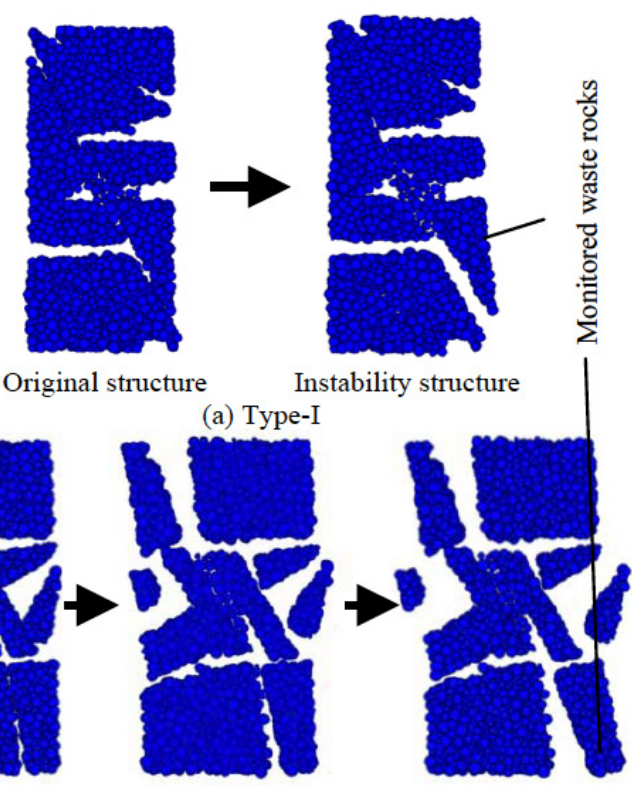

Original structure

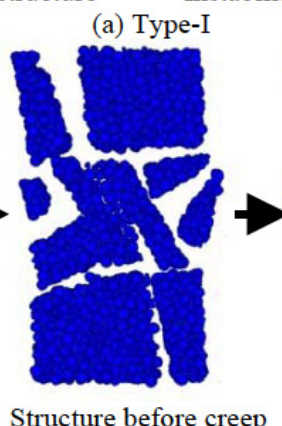

(b) Type-II

Fig. (4). Changes of internal structure of two models.
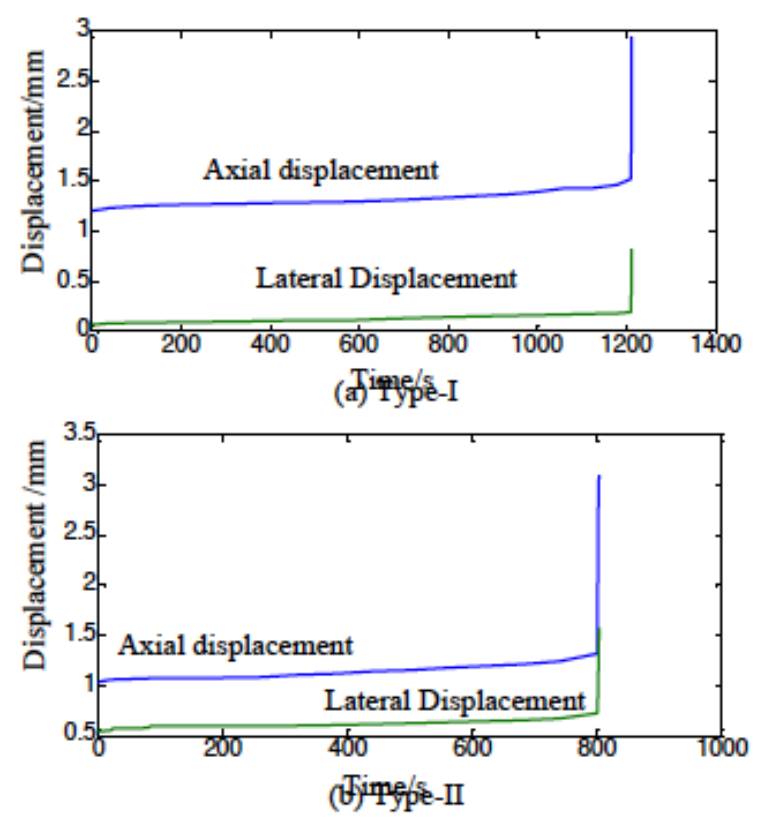

Fig. (5). The displacement curves of stones. 


\section{NON-STATIONARY CREEP MODEL OF CE- MENTED WASTE ROCK BACKFILLS}

In this paper, the Nishihara model comprising a elastic element, two viscoelastic elements and a viscoplastic element as shown in Fig. (6) was taken as the creep model of cemented waste rock backfills. Two viscoelastic elements were used to respectively describe the creep deformation uninfluenced by the waste rock movement and the creep deformation influenced by the waste rock movement. In the Fig. (6), $E_{1}, E_{2}$ and $E_{3}$ are elastic moduli respectively corresponding to the elastic elements. $\eta_{2}, \eta_{3}$ and $\eta_{4}$ are viscosity coefficients respectively corresponding to viscosity elements. $\sigma_{\mathrm{f}} \sigma_{f}$ was the yield limit of the viscoplastic element. For the Nishihara model, the creep equation is shown in formula (2) [13]:

$$
\left\{\begin{array}{l}
\varepsilon=\frac{\sigma}{E_{1}}+\frac{\sigma}{E_{2}}\left(1-e^{-\frac{E_{2}}{\eta_{2}} t}\right) \\
+\frac{\sigma}{E_{3}}\left(1-e^{-\frac{E_{3}}{\eta_{3}} t}\right) \\
\varepsilon=\frac{\sigma}{E_{1}}+\frac{\sigma}{E_{2}}\left(1-e^{-\frac{E_{2}}{\eta_{2}} t}\right) \\
+\frac{\sigma}{E_{3}}\left(1-e^{-\frac{E_{3}}{\eta_{3}}}\right)+\frac{\sigma-\sigma_{f}}{\eta_{4}} t
\end{array}\right.
$$

where $\sigma$ and $\varepsilon$ are loads applied to the Nishihara model and strain of the model generated under the load

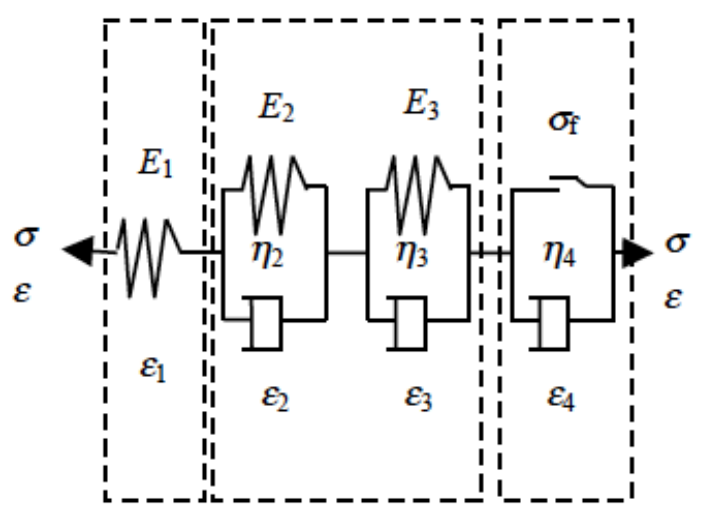

Fig. (6). The creep model.

Because the traditional Nishihara model treats mechanical parameters steadily, it is impossible to describe the deformation characteristics of the material accelerated creep stage. Meanwhile, by observing changes in the internal waste rock structure in the model during the simulation process, it can be seen that the generation and expansion of microcracks in cemented waste rock backfills made the internal waste rock move, thus affecting deformation of cemented waste rock backfills. Therefore, this paper conducted nonstationary treatment of viscoelastic and viscoplastic elements that represented creep deformation under the influence of waste rock movement in the Nishihara model, in order to better describe the creep curves of cemented waste rock backfills.

In the first place, the viscosity coefficient $\eta_{3}$ in the viscoelastic element that represented creep deformation under the influence of waste rock movement was set as the time function shown in Formula (3):

$\eta_{3}(t)=\eta_{30} t^{1-\alpha}$

where $\eta_{30}$ and $\alpha$ are constants.

After the non-stationary viscosity coefficient was replaced to be stationary viscosity coefficient, the nonstationary viscosity element creep compliance was obtained as shown in Formula (4) [14]:

$J_{3}(t)=E_{3}^{-1}\left(1-\exp \left(-\frac{E_{3}}{\eta_{30} \alpha} t^{\alpha}\right)\right)$

Then, the viscosity coefficient $\eta_{4}$ in the viscoplastic element was set as the time function shown in Formula (5):

$\eta_{4}(t)=\eta_{40} e^{-k t}$

where $\eta_{40}$ and $k$ are constants.

After the non-stationary viscosity coefficient was replaced to be stationary viscosity coefficient, the nonstationary viscosity element creep compliance was obtained as shown in Formula (6) [15]:

$J_{4}(t)=\frac{1}{\eta_{40}} t e^{k t}$

Finally, non-stationary creep equation was expressed by the following Formula (7):

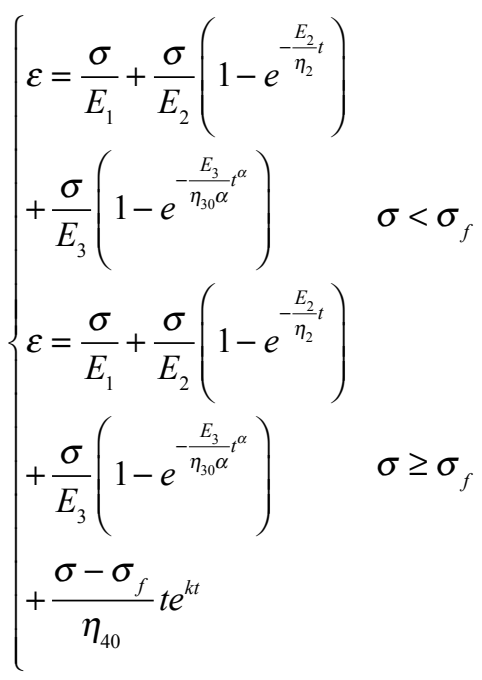

\section{CONCLUSION}

(1) According to the destruction mode, cemented waste rock backfills were divided into two types: slip instability type and tensor instability type. Corresponding to two types of cemented waste rock backfill specimens, PFC was used to establish the corresponding model and creep simulation was implemented. The simulation results showed 
that, under the constant load, waste rocks in the cemented waste rock backfill models more or less moved obviously. The reason causing tensor instability type cemented waste rock backfills to have significant expansion was that the inner waste rocks would severely open externally under the constant load, thus resulting in greater lateral displacement.

(2) Combining the simulation results, the non-stationary cemented waste rock backfill creep model was established to describe the creep characteristics of cemented waste rock backfill.

\section{CONFLICT OF INTEREST}

The authors confirm that this article content has no conflict of interest.

\section{ACKNOWLEDGEMENTS}

This work is supported by the National Natural Science Fund, China (No. 51304083), the national science and technology support program (No. 2013BAB02B08), and Jiangxi province science and Technology Agency Technical Support Plan (No. 20132BBG70106).

\section{REFERENCES}

[1] J. Xu, J. Zhang, Y. Huang, et al, "Experimental research on the compress deformation characteristic of waste-flyash and ite application in backfilling fully mechanized coal mining technology," Journal of Mining \& Safety Engineering, vol. 28, no. 1, pp. 158$162,2011$.

[2] G.-C. He, Y. Liu, D. Ding, Z.-J. Zhang, "Strength characteristic of cemented waste rock backfills and its application," Journal of Mining \& Safety Engineering, vol. 30, no. 1, pp. 74-79, 2013.

[3] Z. Li, "Rheological model and experimental study on high performance concrete with ultra-fine fly ash," unpublished.
[4] P. A. Cundall, and O. D. Strack, "A discrete numerical model for granula assemblies," Geotechnique, vol. 29, no. 1, pp. 47-65, 1979.

[5] C. Zhang, X. Zhan, and C. Yang, "Mesoscopic simulation of strength and deformation characteristics of coarse grained materials," Rock and Soil Mechanics, vol. 34, no. 7, pp. 2077-2083, 2013.

[6] X. Liu, and C. Dong, "Research on Mechanical Characteristics of the Cemented Sandstone Based on 3Dimensional PFC Numerical Model," Journal of Chongqing University, vol. 36, no. 2, pp. 37-44, 2013.

[7] L. X. Cao, Z. Zhou, H. Ye, H. Wu, "Energy dissipation of highstress hard rock with excavation disturbance," Journal of Central South University (Science and Technology), vol. 45, no. 8, pp. 2761-2767, 2014.

[8] Itasca Consulting Group. PFC2D (particle flow code in 2 dimensions) fish in PFC2D, Minneapolis, USA: Itasca Consulting Group, 2008.

[9] J. Sun, M. Chen, Q. Jiang, W.-B. Lu, C.-B. Zhou, "Numerical simulation of mesomechanical characteristics of creep damage evolution for Jingping marble," Rock and Soil Mechanics, vol. 34, no. 12, pp. 3601-3608, 2013.

[10] H. Lee, and S. Jeon, "An experimental and numerical study of fracture coalescence in pre-crached specimens under uniaxial compression," International Journal of Solids and Structures, vol. 48, pp. 979-999, 2001.

[11] D. Huang, D. Cen, and R. Huang, "Influence of medium displacement rate on sandstone with a single pre-crack under uniaxial compression using PFC simulation," Rock and Soil Mechanics, vol. 34, no. 2, pp. 535-545, 2013.

[12] H. Yu, H. Ruan, and W. Chu, "Mesoscopic simulation study of brittle-ductile-plastic transition character of marble," Chinese Journal of Rock Mechanics and Engineering, vol. 32, no. 1, pp. 5564, 2013.

[13] X. Pan, Z. Yang, and J. Xu, "Application study of nonstationary nishihara viscoelasto-plastic rheological model," Chinese Journal of Rock Mechanics and Engineering, vol. 30, no. 1, pp. 2640-2646, 2011.

[14] L. Xiong, L. Yang, and Y. Zhang, "Non-stationary Burgers model for rock," Journal of Central South University (Science and Technology), vol. 41, no. 2, pp. 680-684, 2010.

[15] R. Luo, H. Ruan, Y. Sun, C.-X. Zhu, "Non-stationary parameter creep constitutive model of rocks," Journal of Guilin University of Technology, vol. 27, no. 2, pp. 200-203, 2007.

(C) Li et al.; Licensee Bentham Open.

This is an open access article licensed under the terms of the (https://creativecommons.org/licenses/by/4.0/legalcode), which permits unrestricted, noncommercial use, distribution and reproduction in any medium, provided the work is properly cited. 